\title{
Gli ultraottantenni con malattia renale cronica: una marea montante che ci sommergerà?
}



Dott.ssa Lucia Del Vecchio

Divisione di Nefrologia,

Dialisi, e Trapianto Renale

Ospedale A. Manzoni, Lecco

1.delvecchio@ospedale.lecco.it

Ai suoi albori, all'inizio degli anni Sessanta, la dialisi era considerata come una terapia di salvataggio, che poteva essere offerta solo a un piccolo numero di pazienti, giovani e ben selezionati. Negli anni successivi, sia il miglioramento tecnologico della tecnica dialitica, grazie a innovazioni sostanziali nelle apparecchiature e nei filtri, sia la possibilità di creare accessi vascolari in modo relativamente facile, hanno consentito l'applicazione della tecnica dialitica su larga scala. Questo ha portato alla progressiva estensione dell'indicazione al trattamento dialitico a gruppi di pazienti sempre più anziani e affetti da un numero crescente di comorbidità severe e/o con un'aspettativa di vita limitata.

La dialisi è una terapia salvavita: negare o prescrivere la terapia dialitica ha quindi implicazioni etiche enormi. Negli anni Sessanta questo significava decidere quale paziente potesse beneficiare del trattamento non sulla base delle condizioni cliniche del paziente ma se e dove il trattamento era disponibile.

Oggi nei Paesi industrializzati i Centri Dialisi hanno una diffusione capillare e il trattamento non viene negato a nessuno per motivi organizzativi o economici. Tuttavia il problema etico non è scomparso, ma si è trasformato ed è diventato forse ancora più complesso. In assenza di dati scientifici certi, oggi il nefrologo si trova davanti nel quotidiano alla difficile decisione se e quando un paziente anziano è idoneo al trattamento dialitico, basandosi su dati clinici, su una vaga stima della prognosi, e su inevitabili considerazioni organizzative/assistenziali. Il nefrologo si trova nel mezzo di due forze con direzione opposta. Da un lato in un'epoca di recessione finanziaria sempre più i nostri sistemi sanitari spingono verso una razionalizzazione della spesa sanitaria. La dialisi e i suoi costi vengono sempre più considerati come un lusso non sostenibile in pazienti con un'aspettativa di vita molto ridotta. Dall'altra parte, i successi della medicina ottenuti nell'ultimo secolo hanno portato a una modifica sostanziale delle aspettative dell'uomo nei suoi confronti, che spesso fatica ad accettarne i limiti e gli insuccessi. Di fronte alla possibilità di un trattamento salvavita, molto spesso l'entourage familiare del paziente si trova impreparato a rinunciarvi e spinge al trattamento anche ove il buon senso clinico del nefrologo suggerirebbe diversamente. Tuttavia il buon senso clinico non basta. La decisione al trattamento implica un giudizio inevitabile sul valore e sulla qualità della vita di un paziente. In tutto questo il nefrologo è solo e, soprattutto in Italia, non riceve nessun tipo di formazione specifica, che possa in qualche modo sostenerlo in questo terreno "spinoso".

\section{Dati epidemiologici}

Nelle ultime decadi si è osservato un aumento considerevole e progressivo dei pazienti sia incidenti che prevalenti che richiedono il trattamento dialitico sostitutivo. Questo è andato di pari passo con un progressivo aumento dell'età dei pazienti ammessi alla dialisi. Nel 2008 
i dati del Registro Italiano di Dialisi e Trapianto riportano un'età mediana intorno ai 70 anni nella popolazione dialitica del nostro Paese; circa $1 / 4$ dei pazienti ha un'età vicina o superiore agli ottant'anni (1). Negli Stati Uniti, il tasso d'incidenza dei pazienti in dialisi con più di 75 anni è circa tre volte superiore di quello della fascia d'età compresa tra i 45 e i 64 anni (2). I dati sono ancora più sorprendenti se si considerano solo gli ottuagenari e i nonagenari che iniziano la dialisi. Negli Stati Uniti queste due fasce d'età sono raddoppiate dal 1996 al 2003, con un aumento medio anno del 10\% (3). Se già nel 1999 nei Paesi europei circa la metà dei pazienti che iniziavano la dialisi aveva un'età maggiore di 65 anni (4), il report del 2006 del registro dell'ERA-EDTA indica che il tasso d'incidenza aggiustata di pazienti con 75 anni o più è circa la metà di quella osservata negli Stati Uniti (5), suggerendo importanti differenze sia nelle caratteristiche dei pazienti che nei criteri di accettazione alla dialisi nei diversi Paesi.

Questo è confermato da un recente lavoro contente dati epidemiologici sui pazienti anziani inclusi nel Dialysis Outcomes and Practice Patterns Study (DOPPS) (6). In tutti i Paesi l'età media basale dei pazienti è aumentata nel tempo. Tuttavia la proporzione di pazienti anziani con 75 anni o più varia significativamente nei singoli $\mathrm{Pa}-$ esi, andando dal 40,7\% in Belgio al 16,6\% in Giappone. L'Italia si trova in una situazione intermedia $(23,9 \%)$, simile a quella di Stati Uniti $(23,6 \%)$ e del Canada (24\%).

Diversi fattori rendono probabilmente conto di queste importanti variazioni epidemiologiche osservate nella popolazione dialitica nelle ultime decadi. Sicuramente l'invecchiamento progressivo della popolazione generale e la migliorata sopravvivenza ad altre patologie, come le neoplasie e le malattie cardiovascolari, hanno giocato un ruolo importante. Oltre a ciò, il trattamento dialitico è stato progressivamente esteso a categorie di pazienti con un numero crescente di comorbidità, ai quali un tempo il trattamento era negato. Un chiaro esempio di questo fenomeno sono i pazienti con diabete. Negli anni Settanta solo pochi venivano avviati al trattamento; oggi rappresentano circa la metà dei pazienti che ricevono dialisi negli Stati Uniti. Il fenomeno è inoltre amplificato dal fatto che gli anziani, soprattutto se affetti da comorbidità, hanno ridotto o nessun accesso al trapianto renale rispetto ai pazienti più giovani.

Il progressivo aumento del numero di pazienti ammessi al trattamento dialitico sostitutivo rappresenta solo la punta dell'iceberg della malattia renale cronica dal punto di vista epidemiologico. Dati del programma Third National Health and Nutrition Examination Survey (NHANES III) hanno mostrato un aumento della prevalenza di malattia renale cronica del 15,9\% nella popola- zione americana adulta dal 1988-1994 al 1999-2004 (7). Analizzando i dati per fascia di età, si evidenziava che la malattia renale cronica era più frequente in chi aveva 60 o più anni $(39,4 \%)$ rispetto alle fasce di età più giovani (12,6\% tra i 40 e 59 anni, $8,5 \%$ tra i 20 e i 39 anni).

In epoca recente, l'introduzione routinaria della stima del filtrato glomerulare nella stampa degli esami di laboratorio sembra avere ulteriormente amplificato questi dati epidemiologi, portando a identificare fino al 50\% dei soggetti anziani come affetti da malattia renale cronica (8-11) e, conseguentemente, aumentando in modo incontrollato la richiesta di valutazione e follow-up nefrologico presso i nostri ambulatori. Sembrerebbe tuttavia che tale riduzione del filtrato glomerulare sia clinicamente rilevante solo in una certa percentuale di pazienti (8). Degna d'interesse l'osservazione che i pazienti di età più avanzata, e in particolare gli ultra-ottantenni, sembrerebbero avere una velocità di decadimento del GFR superiore rispetto a pazienti anziani di età inferiore (7). L'impatto di queste osservazioni sull'epidemiologia dei soggetti anziani che arriva o inizia il trattamento dialitico sostitutivo è ancora ignota.

Il ruolo delle comorbidità: quale impatto sulla qualità di vita e prognosi negli ultra-ottantenni con malattia renale cronica?

L'età di per sé è un fattore demografico importante, che condiziona la mortalità dei pazienti con malattia renale cronica stadio V. A questo si aggiunge che i pazienti di età avanzata sviluppano la malattia renale cronica a causa di diabete e/o ipertensione, che ne amplificano il rischio cardiovascolare. Rispetto alla popolazione generale di pari età, i pazienti anziani prevalenti in dialisi hanno una mortalità sei volte superiore (2). L'aspettativa di vita si riduce ulteriormente nei pazienti con più di 80 anni, soprattutto in presenza di comorbidità. La sopravvivenza mediana dei pazienti ottuagenari o nonagenari che iniziano dialisi negli Stati Uniti è compresa tra solo 8 e 15 mesi ed è nettamente inferiore a quella dei soggetti di pari età nella popolazione generale (3).

I dati dello studio DOPPS mostrano, nei pazienti con 75 anni o più, una sopravvivenza mediana di 3,3 anni in Europa, 5,4 anni in Giappone e 2,5 anni in Nord America (6). La relazione tra età e rischio di morte è complessa, non solo per queste significative differenze tra i singoli Paesi, ma anche perché la differenza di rischio tra pazienti anziani e pazienti più giovani è più evidente in alcuni regioni (come Europa e Giappone) rispetto a Australia, Nuova Zelanda e Nord America (6).

Oltre all'età, razza e malattia di base, la presenza di comorbidità e/o disabilità giocano un ruolo preponderan- 
te nell'influenzare il rischio di morte dei pazienti anziani in dialisi.

Ad esempio, i dati dello studio DOPPS indicano una prevalenza di malattia coronarica tre volte maggiore nei pazienti con più di 75 anni rispetto a chi ne ha meno di 45 anni (6).

Anche il diabete ha un ruolo importante. Come è ben noto la sopravvivenza dei diabetici in dialisi è inferiore a quella dei non diabetici. Tuttavia nei pazienti anziani sembrerebbe che la differenza in termini di sopravvivenza rispetto ai soggetti di pari età della popolazione generale sia meno marcata quando paragonata alle fasce d'età più giovani (8).

La cachessia è un'importante causa di morte nei pazienti anziani che ricevono il trattamento dialitico sostitutivo (9). Tuttavia non è ben chiara la relazione di causa-effetto, dato che molte patologie concomitanti possono causare malnutrizione e morte. Partendo dall'ipotesi che un'infiammazione cronica su base aterosclerotica possa avere un ruolo importante nel determinare malnutrizione e morte nei pazienti con malattia renale cronica avanzata, è stata coniata la definizione di sindrome MIA (malnutrizione, infiammazione e aterosclerosi) (10). La malnutrizione riduce le difese immunitarie, predisponendo allo sviluppo d'infezioni, soprattutto nei pazienti anziani, spesso portatori di catetere venoso centrale o allettati, e quindi con ridotta ventilazione polmonare e a rischio di sviluppare polmoniti.

Il problema nutrizionale ha anche risvolti etici inevitabili, spesso di difficile soluzione, che vanno di pari passo con l'eventuale inizio di un trattamento dialitico sostitutivo.

La capacità di camminare è una misura di performance importante dei pazienti in dialisi, che ha anche implicazioni prognostiche. Negli Stati Uniti circa la metà dei pazienti in dialisi con più di 65 anni ha qualche difficoltà nel camminare, il 10\% è allettato o in carrozzina (2). Il rischio di morte in questi pazienti aumenta in presenza di difficoltà alla deambulazione e raggiunge il suo massimo in chi non è in grado di camminare (2). La demenza e un pregresso ictus sono le due comorbidità più spesso associate alle difficoltà di deambulazione.

Come nella popolazione generale, la presenza di demenza, contribuisce a influenzare negativamente la prognosi dei pazienti anziani in dialisi e ad influenzarne negativamente la qualità della vita. Un'elevata percentuale di pazienti in dialisi ha un deficit cognitivo (11), spesso non riconosciuto (12). Dati provenienti dallo studio DOPPS hanno evidenziato che solo il $4 \%$ dei pazienti ha una diagnosi riconosciuta di demenza; dopo aggiustamento per fattori confondenti essa si associa a un aumento significativo del rischio di morte (16).
Questo sembra essere vero anche per chi ha un deficit cognitivo modesto (13).

Tutto ciò espone il paziente anziano in dialisi a un elevato rischio di caduta, con un ulteriore peggioramento della prognosi (14). Chi ha cadute infatti ha un rischio doppio di morte e di ospedalizzazioni e 3,5 volte più elevato di essere ammesso in una struttura assistita (15).

\section{Qual è l'impatto della dialisi sull'aspettativa di vita degli ultra-ottuagenari?}

Sfortunatamente solo pochi studi hanno paragonato la sopravvivenza dei pazienti anziani in dialisi rispetto a chi veniva mantenuto in terapia conservativa. Sembrerebbe che chi viene ammesso al trattamento dialitico sostitutivo sopravviva più a lungo, ma inevitabilmente questi studi sono influenzati da grossi bias di selezione dei pazienti. Murtagh et al (16) hanno valutato la sopravvivenza di 129 pazienti con più di 75 anni nell'anno successivo alla decisione d'iniziare o meno il trattamento dialitico sostitutivo. In questo periodo, in linea con altre casistiche, sono deceduti il $23 \%$ dei pazienti in dialisi e ben il $66 \%$ dei pazienti per i quali si era optato per il trattamento conservativo. Tuttavia, il vantaggio in termini di sopravvivenza del trattamento dialitico si riduceva notevolmente in presenza di comorbidità severe. Analogamente, uno studio francese su 146 ottuagenari con malattia renale cronica stadio $\mathrm{V}$ ha confermato che chi inizia la dialisi vive più a lungo di chi non la inizia, con una sopravvivenza mediana di 28,9 e 8,9 mesi, rispettivamente. Fattori indipendenti in grado di predire il decesso erano la malnutrizione, la presa in carico tardiva da parte del nefrologo, e il grado d'incapacità funzionale; la gravità delle comorbidità non era significativamente diversa nei due gruppi.

Chi inizia la dialisi sembrerebbe essere gravato da un maggior numero di ospedalizzazioni, mentre chi viene avviato al trattamento conservativo ha una probabilità maggiore di morire a casa o in hospice (17).

Infine, l'inizio della dialisi nei pazienti anziani con malattia renale cronica stadio $\mathrm{V}$ coincide con un rapido declino delle capacità funzionali, come dimostrato da uno studio di registro di grandi dimensioni condotto su più di 3000 soggetti provenienti da case di riposo americane (18).

\section{Quando e se iniziare la dialisi negli ottuagenari}

Dati gli elevati tassi di mortalità e i costi del trattamento dialitico sostitutivo, considerazioni etiche sono doverose nel bilanciare i possibili benefici del trattamento da un lato con la prognosi a breve e lungo termine e la qua- 
lità della vita del paziente. Il nefrologo ha il dovere di proporre tutte le opzioni terapeutiche possibili ove ragionevole. Purtroppo il limite di ciò che è ragionevole è molto sfumato e non può prescindere da considerazioni soggettive. A volte il limite della ragionevolezza viene posto a priori dal medico curante, che non ritiene neppure indicato inviare il paziente al nefrologo (19). A mio parere, e come peraltro testimoniato dai dati della letteratura, questo è sicuramente un errore, poiché la decisione di non iniziare il trattamento dialitico sostitutivo non implica il nichilismo terapeutico assoluto, bensì l'attuazione di una terapia conservativa ben calibrata che solo il nefrologo può e deve attuare nelle fasi più avanzate della CKD.

Tra le ragioni che pesano di più nella decisione di non iniziare il trattamento dialitico sostitutivo troviamo l'età avanzata, un deficit neurologico, l'insufficienza terminale di altri organi (cuore, fegato in primis), la presenza di neoplasie metastatizzate, comorbidità multiple e, non ultimo, il rifiuto da parte del paziente e/o della famiglia di effettuare il trattamento.

La presenza di una demenza severa è considerata come uno dei principali criteri di esclusione dal trattamento. Tuttavia anche in questo caso, all'apparenza semplice, le implicazioni etiche sono complesse, poiché non è cosi ovvio comprendere il grado di felicità e la qualità della vita di una persona con demenza moderata o severa (20). Qualche anno fa, il French Renal Epidemiology and Information Network (REIN) ha proposto uno score clinico che potrebbe essere di aiuto nel predire la prognosi del paziente anziano che inizia la dialisi (21). Tuttavia i predittori clinici vanno usati con estrema cautela, poiché spesso il singolo paziente esce dagli schemi generali della statistica.

Oltre al giudizio clinico, differenze culturali e di formazione possono giocare un ruolo, rendendo conto in parte anche della diversa attitudine a iniziare il trattamento riscontrate in Paesi differenti (22).

Nel 2000 la Renal Physicians Association e l'American Society of Nephrology hanno prodotto delle linee guida per guidare i nefrologi nella difficile decisione di iniziare o interrompere il trattamento dialitico sostitutivo (23). Dopo 10 anni è stata pubblicata la revisione di queste linee guida (24) che, a titolo di provocazione, invitano il medico a porsi il seguente quesito: «Rimarrei sorpreso se questo paziente morisse nel prossimo anno?» Non solo. Nel documento viene discusso il ridotto riconoscimento e trattamento del dolore e dei sintomi del paziente uremico, insieme alla sottoutilizzazione di hospice nei pazienti dializzati. Infine viene ribadita la necessità di concordare un obiettivo di cura del paziente con gradi di aggressività commisurati alle condizioni cliniche complessive.
Il nefrologo però non solo deve affrontare la difficile decisione se iniziare o meno la dialisi in un dato paziente, ma anche quando questa debba essere fatta e come. Purtroppo non esistono indicazioni chiare in merito.

A fronte di una progressiva tendenza negli ultimi anni d'iniziare la dialisi sempre più precocemente, anche nell'anziano, studi recenti sembrano supportare la mancanza di un reale beneficio di intraprendere precocemente il trattamento dialitico sostitutivo (25).

Alla luce di questi dati e del fatto che l'impatto del trattamento dialitico nell'anziano è spesso poco prevedibile, sembra ragionevole suggerire di procrastinare il più possibile l'inizio del trattamento mediante terapia di supporto.

In merito a ciò, uno studio randomizzato condotto nel nostro Paese su 112 pazienti con più di 70 anni e malattia renale cronica stadio V (GFR tra 5 e $7 \mathrm{ml} / \mathrm{min} / 1,73 \mathrm{~m}^{2}$ ) ha dimostrato una sopravvivenza analoga in chi veniva randomizzato ad iniziare il trattamento dialitico sostitutivo rispetto a chi riceveva una dieta ipoproteica (25). Va comunque considerato che circa i due terzi dei pazienti randomizzati alla dieta ipoproteica hanno iniziato in seguito il trattamento dialitico sostitutivo per sovraccarico idrosalino e/o iperkaliemia. Sorge la domanda se in questi pazienti non sarebbe stato sufficiente una dieta ipoproteica meno spinta (e quindi con un rischio inferiore di malnutrizione) per procrastinare il più possibile il trattamento dialitico sostitutivo. Non sappiamo inoltre se questi risultati interessanti possano essere applicati anche a pazienti con severe comorbidità, poiché esclusi dal trial.

La dialisi peritoneale è una valida alternativa all'emodialisi nel paziente anziano (26), spesso sottoutilizzata però per bias, sia di prescrizione, che di difficoltà oggettive dei familiari nel gestire la metodica. È però auspicabile nel prossimo futuro una sua implementazione nei pazienti uremici degenti nelle case di riposo ove è già presente personale sanitario addestrato, che potrebbe farsi carico della gestione del trattamento in questi pazienti.

Infine, un aspetto da non dimenticare nell'approccio al problema dialisi nell'anziano è la gestione dell'accesso vascolare. Il paziente anziano presenta spesso un albero vascolare inadeguato. L'allestimento della fistola artero-venoso dovrebbe essere confezionata con largo anticipo, per consentirne un'adeguata maturazione. D'altro canto però, la procedura chirurgica di per sé espone il paziente ad ospedalizzazione, possibile aggravamento delle comorbidità, peggioramento della demenza e possibile sviluppo d'infezione. Inoltre, la scarsa aspettativa di vita, legata sia all'età che alla situazione clinica, comporta il rischio che il paziente 
possa morire prima d'iniziare la dialisi e quindi di essersi sottoposto inutilmente a un intervento chirurgico per l'allestimento dell'accesso vascolare (27). Anche il ricorso sempre più frequente ai cateteri venosi centrali non appare come soluzione ottimale, per il rischio d'infezioni e sotto-dialisi.

\section{Abbiamo il coraggio d'interrompere quanto già iniziato?}

L'interruzione del trattamento dialitico sostitutivo è relativamente comune, soprattutto negli Stai Uniti e in Australia. Dati dell'United States Renal Data System (USRDS) indicano che nella coorte di pazienti prevalenti in dialisi nel 2005-2006, circa un quarto dei soggetti deceduti ha interrotto il trattamento dialitico prima di morire a causa di complicanze mediche o per la definizione generica, tradotta con qualche difficoltà dal termine inglese, di "impossibilità a vivere in modo prospero" (2). In quest'ultima definizione rientrano con buona probabilità i pazienti con quadri severi di cachessia e a volte di demenza.

Dati provenienti da sei Registri europei di dialisi hanno mostrato una mortalità per "cause sociali", che comprende anche l'interruzione del trattamento dialitico sostitutivo, in circa l' $8 \%$ dei pazienti con età compresa tra $i$ 65 e i 74 anni e del 13\% in chi ha 75 anni o più (4).

Il ricorrere all'interruzione del trattamento dialitico è sicuramente influenzato, oltre che dalle caratteristiche cliniche dei pazienti, anche da fattori organizzativi e culturali, come dimostrato da notevoli differenze numeriche nei diversi Paesi del mondo. Una recente pubblicazione di dati dello studio DOPPS (6) ha mostrato che l'interruzione del trattamento dialitico rende conto del
3,5\% dei decessi dei pazienti anziani in Europa, del 14,3\% in Australia e Nuova Zelanda e del 7,6\% negli Stati Uniti. In Giappone non vengono riportati decessi legati all'interruzione del trattamento.

\section{Conclusioni}

Nei pazienti anziani con malattia renale cronica stadio V, morbilità e mortalità sono spesso elevate e la qualità di vita estremamente scarsa. Questo implica inevitabilmente il sorgere di una serie di quesiti etici, legati all'identificazione del limite delle cure che è lecito offrire al paziente, senza sconfinare nell'accanimento terapeutico. D'altro canto è spesso difficile interrompere il trattamento dialitico, una volta che questo è stato iniziato, se le condizioni cliniche e/o neurologiche del paziente si deteriorano.

Purtroppo le linee guida su questi aspetti critici sono ancora insufficienti e comunque possono aiutare solo in parte il clinico nella decisione del trattamento del singolo paziente. Sicuramente l'implementazione di una maggiore formazione culturale su questi aspetti, insieme a una sempre maggiore diffusione al ricorso di una decisione condivisa tra medico e paziente (o entourage familiare) potranno, sia ridurre il ricorso a terapie complesse ove non indicato, che offrire una chance terapeutica a coloro che in qualche modo potrebbero beneficiarne.

L'età avanzata di per sé non può essere considerata una controindicazione al trattamento dialitico sostitutivo, ma la coesistenza di comorbidità severa, oltre all'età avanzata, deve fare riflettere se il trattamento dialitico rappresenti davvero la migliore opzione possibile.

\section{Bibliografia}

1. http://www.sin-ridt.org/Italia/Report2008/REPORT\%20 RIDT\%202008\%20NOTE.pdf

2. Collins AJ, Foley RN, Herzog C, et al. United States Renal Data System 2008 Annual Data Report Abstract. Am J Kidney Dis 2009; 53(1 Suppl): vi-vii, S8-374.

3. Kurella M, Covinsky KE, Collins AJ, Chertow GM. Octogenarians and nonagenarians starting dialysis in the United States. Ann Intern Med 2007; 146(3): 177-83.

4. Jager KJ, van Dijk PC, Dekker FW, Stengel B, Simpson K, Briggs JD; ERA-EDTA Registry Committee. The epide-

mic of aging in renal replacement therapy: an update on elderly patients and their outcomes. Clin Nephrol 2003; 60(5): 352-60.

5. European Dialysis and Transplant Association Registry. 2006 Annual Report. July 2008. Available at http://www. era-edta-reg.org/index.jsp?p=annrep

6. Canaud B, Tong L, Tentori F, et al. Clinical Practices and Outcomes in Elderly Hemodialysis Patients: Results from the Dialysis Outcomes and Practice Patterns Study (DOPPS). Clin J Am Soc Nephrol 2011; 6(7): 1651-62. 
7. Centers for Disease Control and Prevention (CDC). Prevalence of chronic kidney disease and associated risk factors-United States, 1999-2004. MMWR Morb Mortal Wkly Rep 2007; 56(8): 161-5.

8. Nitsch D, Burden R, Steenkamp R, et al. Patients with diabetic nephropathy on renal replacement therapy in England and Wales. QJM 2007; 100(9): 551-60.

9. Jager KJ, van Dijk PC, Dekker FW, Stengel B, Simpson K, Briggs JD; ERA-EDTA Registry Committee. The epidemic of aging in renal replacement therapy: an update on elderly patients and their outcomes. Clin Nephrol 2003; 60(5): 352-60.

10. Stenvinkel P, Heimburger O, Paultre F, et al. Strong association between malnutrition, inflammation, and atherosclerosis in chronic renal failure. Kidney Int 1999; 55: 1899-911.

11. Murray AM, Tupper DE, Knopman DS, et al. Cognitive impairment in hemodialysis patients is common. Neurology 2006; 67 (2): 216-23.

12. Kurella M, Mapes DL, Port FK, Chertow GM. Correlates and outcomes of dementia among dialysis patients: the Dialysis Outcomes and Practice Patterns Study. Nephrol Dial Transplant 2006; 21(9): 2543-8.

13. Griva K, Stygall J, Hankins M, Davenport A, Harrison M, Newman SP. Cognitive impairment and 7-year mortality in dialysis patients. Am J Kidney Dis 2010; 56(4): 693-703.

14. Abdel-Rahman EM, Turgut F, Turkmen K, Balogun RA. Falls in elderly hemodialysis patients QJM 2011 Jul 12. [Epub ahead of print].

15. Abdel-Rahman EM, Yan G, Turgut F, Balogun RA. Longterm morbidity and mortality related to falls in hemodialysis patients: role of age and gender - a pilot study. Nephron Clin Pract 2011; 118(3): c278-84.

16. Murtagh FE, Marsh JE, Donohoe P, Ekbal NJ, Sheerin NS, Harris FE. Dialysis or not? A comparative survival study of patients over 75 years with chronic kidney disease stage 5. Nephrol Dial Transplant 2007; 22(7): 1955-62.

17. Carson RC, Juszczak M, Davenport A, Burns A. Is maximum conservative management an equivalent treatment option to dialysis for elderly patients with significant comorbid disease? Clin J Am Soc Nephrol 2009; 4(10): 1611-9.

18. Kurella Tamura M, Covinsky KE, Chertow GM, Yaffe K,
Landefeld CS, McCulloch CE. Functional status of elderly adults before and after initiation of dialysis. N Engl J Med 2009; 361(16): 1539-47.

19. Sekkarie MA, Moss AH. Withholding and withdrawing dialysis: the role of physician specialty and education and patient functional status. Am J Kidney Dis 1998; 31(3): 464-72.

20. Spike JP. Responding to requests for dialysis for severely demented and brain injured patients. Semin Dial 2007; 20(5): 387-90.

21. Couchoud C, Labeeuw M, Moranne O, et al.; for the French Renal Epidemiology and Information Network (REIN) registry. A clinical score to predict 6-month prognosis in elderly patients starting dialysis for end-stage renal disease. Nephrol Dial Transplant 2009; 24(5): 1553-61.

22. Lambie M, Rayner HC, Bragg-Gresham JL, et al. Starting and withdrawing haemodialysis-associations between nephrologists' opinions, patient characteristics and practice patterns (data from the Dialysis Outcomes and Practice Patterns Study. Nephrol Dial Transplant 2006; 21(10): 2814-20.

23. Renal Physicians Association and American Society of Nephrology Clinical Practice Guideline. Shared decisionmaking in the withhold and withdrawal from dialysis. Washington DC, 2000, Report No 2.

24. Moss AH. Revised dialysis clinical practice guideline promotes more informed decision-making. Clin J Am Soc Nephrol 2010; 5(12):2380-3.

25. Brunori G, Viola BF, Parrinello G, et al. Efficacy and safety of a very-low-protein diet when postponing dialysis in the elderly: a prospective randomized multicenter controlled study. Am J Kidney Dis 2007; 49(5): 569-80.

26. Lim W, Dogra G, McDonald SP, Brown FG, Johnson DW. Compared with younger peritoneal dialysis patients, elderly patients have similar peritonitis-free survival and lower risk of technique failure, but higher risk of peritonitis-related mortality. Perit Dial Int 2011 May 31. [Epub ahead of print].

27. O'Hare AM, Bertenthal D, Walter LC, et al. When to refer patients with chronic kidney disease for vascular access surgery: should age be a consideration? Kidney Int 2007; 71(6): $555-61$. 